\title{
Leprosy-Related Chronic Arthritis with an Abundance of Mycobacterium Leprae in the Synovial Fluid: A Rare Case
}

\section{Hendra Gunawan (DD \\ Yuli Wulansari \\ Pati Aji Achdiat \\ Reti Hindritiani}

Department of Dermatology and Venereology, Faculty of Medicine, Universitas Padjadjaran - Hasan Sadikin General Hospital, Bandung, West Java, 40I6I, Indonesia
Correspondence: Hendra Gunawan Department of Dermatology and Venereology, Faculty of Medicine, Universitas Padjadjaran-Hasan Sadikin General Hospital, Jl. Pasteur No. 38, Bandung, West Java, 4016I, Indonesia Tel/Fax +62 222032426 Ext. 3449 Email h.gunawan2016@unpad.ac.id

\begin{abstract}
Bone and joint involvements are the most common manifestation in leprosy, after cutaneous and neurological manifestations, yet are underdiagnosed and rarely reported. We report a case of lepromatous leprosy in a 41-year-old male with bone and joint changes. Physical examination revealed a leonine face, madarosis, hypesthetic hypopigmented macules on the back, and swelling of fingers and toes. Acid-fast bacilli from slit-skin smear and synovial fluid in an interphalangeal joint of the hand were found. Radiographic features showed osteolysis and destruction of some phalanges of the hands, bone erosion and destruction of some phalanges of the feet, as well as destruction and subluxation of some hand joints. The patient was given multidrug therapy for multibacillary leprosy, resulting in the reduction of swelling in fingers and toes. In conclusion, the examination of bone and joint changes in leprosy patients is important for early detection and management of musculoskeletal complications, to prevent future deformities and disabilities.
\end{abstract}

Keywords: bone, joint, leprosy, Mycobacterium leprae, synovial fluid

\section{Introduction}

Leprosy is a chronic granulomatous infectious disease caused by Mycobacterium leprae (M. leprae) that most often affects the skin and nerves. ${ }^{1,2,3}$ Following the skin and nerves, bone and joint changes are the third most common manifestation of leprosy. ${ }^{4,5}$ The diagnosis of leprosy can be a problem for clinicians when musculoskeletal features constitute the initial presentation with minimal or even none of the skin or nerves involvement. ${ }^{5}$ Furthermore, musculoskeletal problems in leprosy can resemble other connective tissue disorders, which may lead to misdiagnosis and mistreatment. ${ }^{6}$ Therefore, it is important for clinicians to have good knowledge about several aspects of this condition. ${ }^{5}$ In addition, the examination and early treatment of bone and joint complications can reduce disabilities and deformities in leprosy. ${ }^{1,4,7}$ This case report aimed to show a rare finding of abundant Mycobacterium leprae in the synovial fluid of a lepromatous leprosy patients with bone changes and leprosy-related chronic arthritis.

\section{Case Report}

A 41-year-old Indonesian male came to our outpatient clinic due to swelling in fingers and toes for the last nine months. He had limitations in activities of daily living. There was no history of shortening of the digits and joint stiffness in the mornings. Upon 
physical examination, the patient had leonine facies and madarosis. Hypesthetic hypopigmented macules were found on the right side of the back. (Figure 1) Sensory nerves examination revealed glove and stocking hypesthesia. Fingers and toes were swollen, without redness, warmth, and tenderness on the overlying skin. (Figure 2A) The range of motion was limited in the proximal and distal

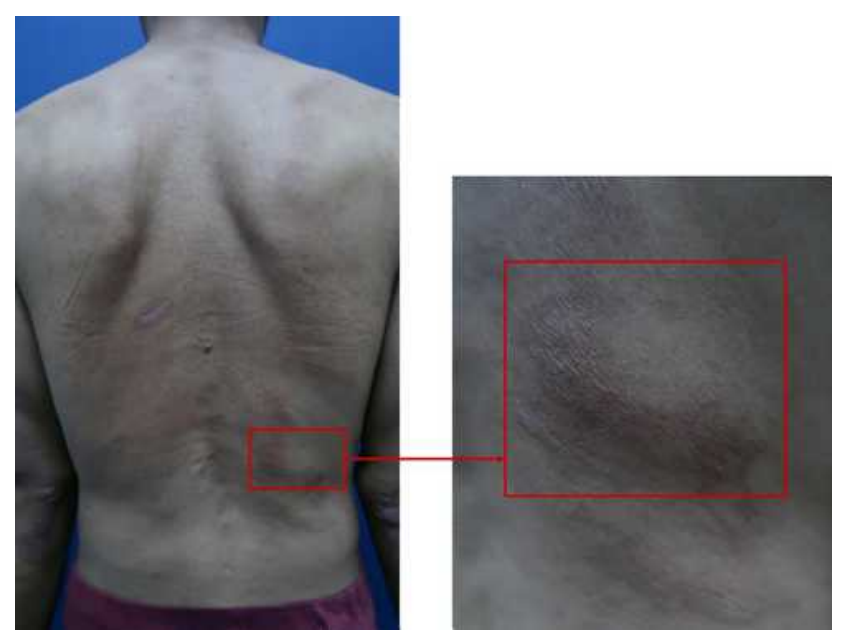

Figure I Presence of hypesthetic hypopigmented macules on the right side of the back. interphalangeal joints of the fingers. Claw fingers, drop wrist, muscle atrophy, or contracture of the hands was not detected. Claw toes, drop foot, muscle atrophy, or contracture of the feet was not found. A slit-skin smear showed a bacterial index of $4.38+$ (Figure 3 ) and a morphological index of $79 \%$. We referred the patient to the Department of Orthopedic and Traumatology to perform synovial fluid aspiration from the interphalangeal joint of the hand (Figure 4A). Acid-fast staining of synovial fluid from the interphalangeal joint of the hand revealed numerous acidfast bacilli (Figure 4B). Histopathological examination on a hypesthetic macule from the back showed epidermal atrophy with Grenz zone. The dermis was minimally infiltrated with lymphocytes and foamy histiocytes, which confirmed the diagnosis of lepromatous leprosy. Radiographic features showed osteolysis and destruction of some phalanges of the hands, bone erosion and destruction of some phalanges of the feet, as well as destruction and subluxation of some hand joints (Figure 2B). These bone changes were concluded as osteomyelitis. The final diagnosis was lepromatous leprosy, osteomyelitis and chronic arthritis due to leprosy, with hand and foot deformities. The patient received multidrug therapy (MDT) for multibacillary (MB) leprosy. Improvements in swollen fingers and toes were observed within two months
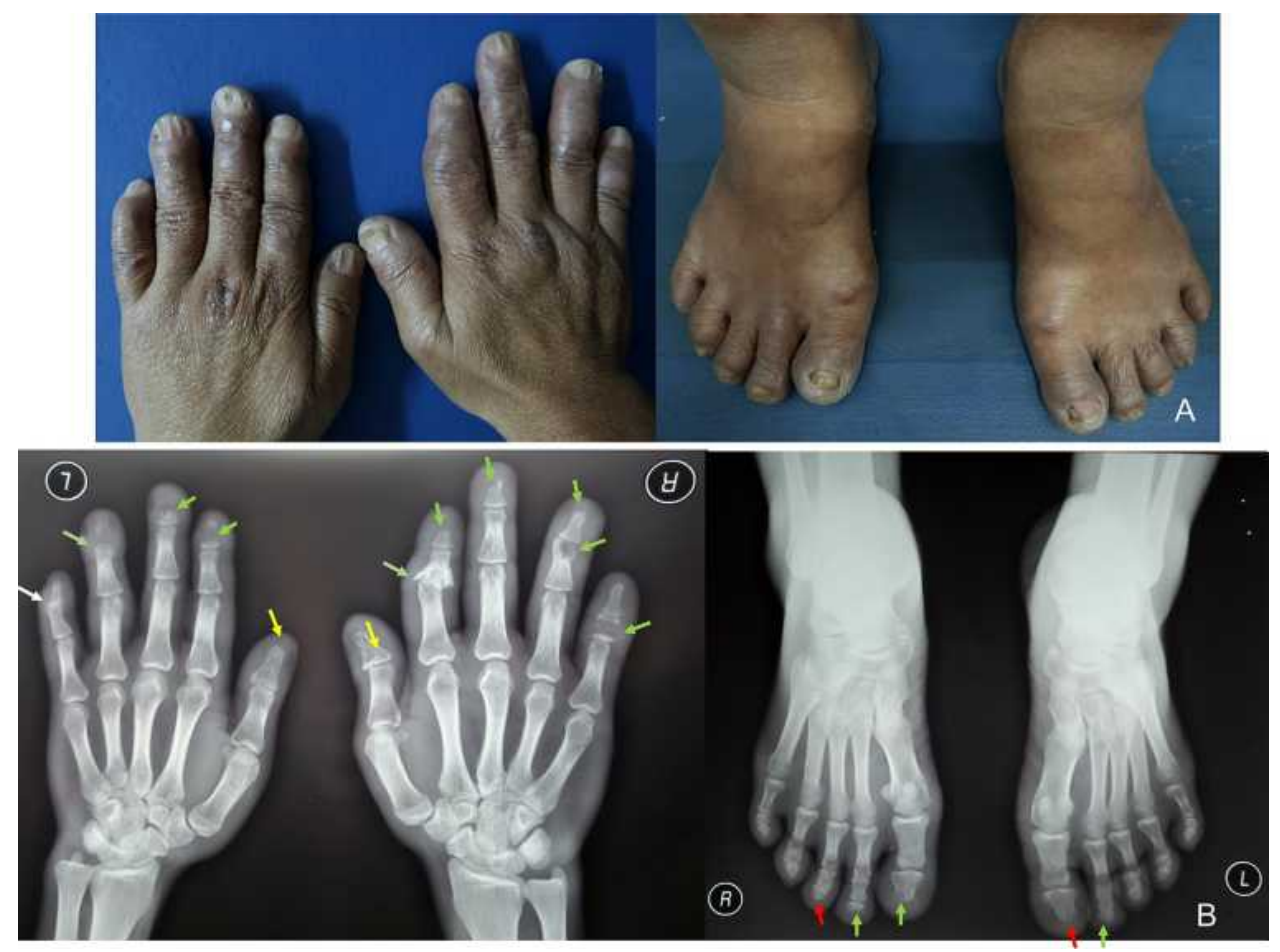

Figure 2 (A) Swollen fingers and toes. (B) Radiographic features showed osteolysis and destruction of some phalanges of the hands, bone erosion and destruction of some phalanges of the feet, and destruction and subluxation of some hand joints. (yellow arrow: osteolysis; green arrow: destruction; red arrow: erosion; white arrow: subluxation). 


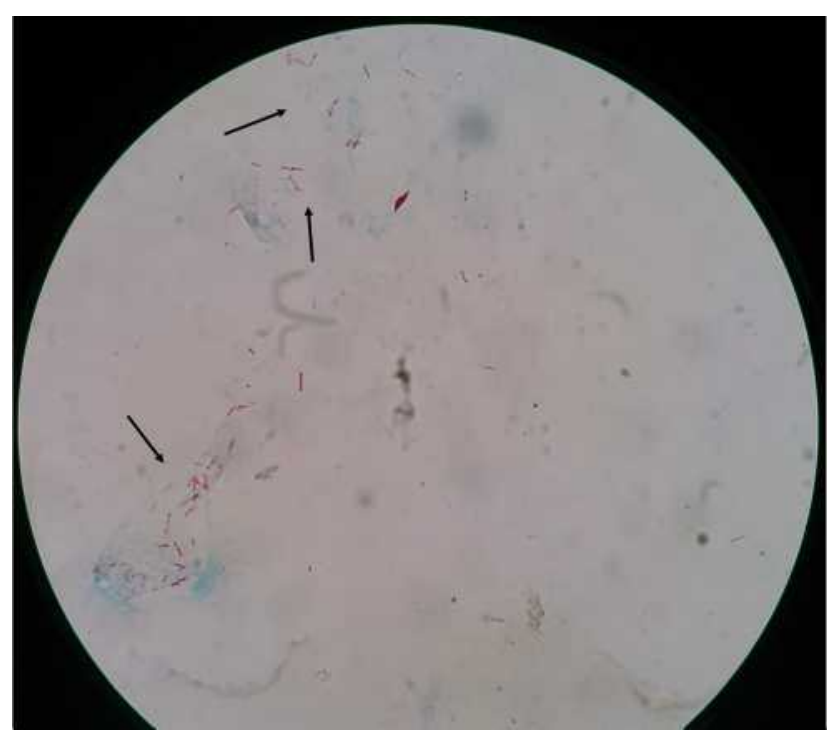

Figure 3 Bacilloscopy of smear showing positive bacillus (black arrow).

after the start of treatment. The patient could move hands more freely to do daily activities. There were no changes on skin lesions.

\section{Discussion}

Leprosy is primarily a health problem and remains a leading cause of deformities and disabilities. ${ }^{1,4}$ Leprosy can manifest an enormous variety of signs and symptoms including arthralgia and arthritis resembling those seen in some rheumatic diseases, often posing diagnostic difficulties in endemic areas. ${ }^{4,8}$ Furthermore, with globalization and increasing travel populations, it is possible that clinics in non-endemic countries may come across cases of leprosy with rheumatological manifestations. ${ }^{3,4}$
Therefore, recognition of bone and joint involvements in leprosy is important to prevent unnecessary diagnostic tests, to establish accurate diagnosis, and to give early treatment. ${ }^{3,8}$

Bone changes are one of the important prognostic factors in leprosy ${ }^{7}$ and can be divided into three groups: specific, non-specific, and osteoporotic bone changes. ${ }^{7,8,10}$ The frequency of bone changes has varied from $15 \%$ to $29 \%$ of leprosy cases. ${ }^{7}$ Specific bone changes are caused by direct actions of $M$. leprae on bone tissues. ${ }^{1,7,11}$ Specific bone changes in leprosy are rare variants and the prevalence ranges from $3 \%$ to $5 \%$. It primarily affects small bones of the face, hands, and feet. ${ }^{1}$ Non-specific bone changes are far more frequent, nearly twice as much as specific changes. They are classified as neurotrophic lesions and lesions due to superinfections, that may overlap. ${ }^{7}$ Motor denervation is sometimes related to the absorption of cancellous bone and progresses into a concentric type of bone atrophy, which affects the length, the width, or both. The tips of insensitive fingers and toes were affected by changes due to distal absorption. ${ }^{1}$ Progressive absorption with loss of digits and the development of the so-called "mitten hand" occurred when that process is complicated by infections. $1,7,10$

Joint manifestations occur in approximately $75 \%$ of people with leprosy and may represent one of the early symptoms. $^{4,8}$ Proposed mechanisms include reactional states, direct infiltration of the synovium, and peripheral sensory neuropathy leading to joint destruction., ${ }^{4,9,12}$ There is no official classification of arthritis in leprosy. Nevertheless, arthritis in leprosy can be categorized into the following groups: (i) Charcot's joints, (ii) septic arthritis, (iii) acute polyarthritis of lepra reaction, and (iv) chronic
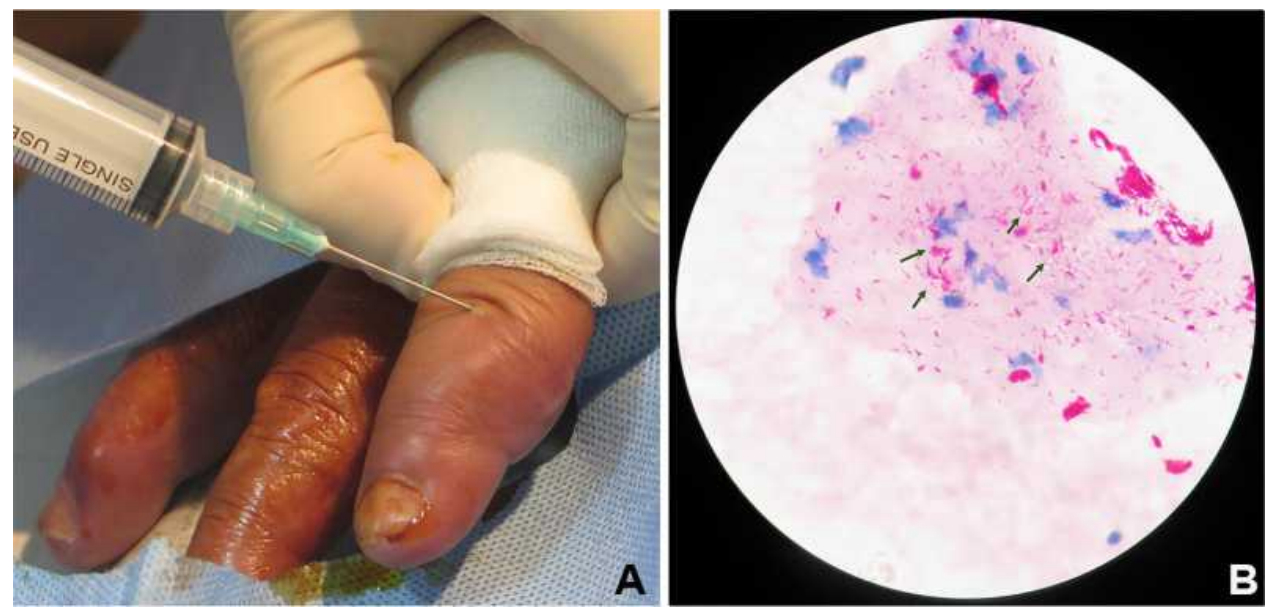

Figure 4 (A) Synovial fluid aspiration from the interphalangeal joint of the hand. (B) Acid-fast staining of synovial material from the interphalangeal joint of the hand showed numerous acid-fast bacilli (black arrow). 
arthritis. ${ }^{4,5,8}$ Charcot's joints, otherwise known as neuropathic arthropathy, manifests as joint dislocations, pathological fractures, and debilitating deformities usually involving the weight-bearing joints of the lower limbs, such as ankles and the knees. ${ }^{4,13}$ Acute arthritis in leprosy usually occurs as part of leprosy reactions. ${ }^{4,8,12}$ Acute arthritis manifests as symmetrical inflammatory polyarthritis affecting small joints of the hands and feet, which resembles rheumatoid arthritis (RA). ${ }^{4,13}$ Knees, ankles, shoulders, and elbows may be affected. Acute arthritis uncommonly manifests as joint destruction. ${ }^{4}$ Arthritis was discovered in $20(65 \%)$ out of 31 patients with leprosy reaction according to a study by Gibson et al. ${ }^{14}$ Atkin et al ${ }^{15}$ reported primarily type I leprosy reaction-associated arthritis in 19 (50\%) patients out of 38 patients. Chronic arthritis could result from bacilli infiltration, involving small joints in symmetrical polyarthritis distribution, affecting primarily the wrists, metacarpals, and proximal interphalangeal joints. ${ }^{4,12}$ As a result, irreversible joint damages can occur, most remarkably in the hands as stated by Atkin et al in their study. ${ }^{16}$ Cossermelli-Messina et al ${ }^{17}$ reported 39 cases of leprosy with arthritis not related to lepra reactions. Arthritis was chronic in most of the patients (mean duration 11 years) and RA-like in distribution.

Medical imaging in leprosy is important to assist the diagnosis of musculoskeletal involvement. ${ }^{1,4,18}$ Specific bone changes causing granulomatous lesions, which are destructive and seen as focal areas of increased rarefaction. ${ }^{1,10}$ They manifest radiographically as primary periostitis, honeycombing, subarticular erosion, enlarged nutrient foramina, concentric cortical erosions, and bone cyst. ${ }^{11}$ Periostitis and sclerosis are signs of the healing process. ${ }^{10}$ The radiographic appearances of non-specific bone changes may indicate resorption of bones in the distal phalanges of the fingers and toes or acroosteolysis. Other non-specific bone changes presentations are spontaneous fractures, dislocations, acute and chronic osteomyelitis. $^{7}$ Radiographic features in leprosy patients with arthritis are variable, ranging from normal joints to joint subluxations and complete destructions. ${ }^{4}$ In this patient, radiographic features showed osteolytic and destruction of some phalanges of both hands as well as erosion and destruction of some phalanges of the feet. These appearances were interpreted as osteomyelitis. The result of radiographic examination in this patient is in accordance with non-specific bone changes in leprosy. In addition to that, we discovered joint destruction and subluxation of the hands from radiographic features, which are signs of arthritis in leprosy.
The gold standard for diagnosis of arthritis due to leprosy remains to be the presence of $M$. leprae bacilli in the joint, despite the difficulty to find leprosy bacilli in the joint. ${ }^{4}$ Arthrocentesis can facilitate the diagnosis of infection within a joint space. Joint aspiration should be performed by a trained clinician under sterile conditions, not only to minimize the risk of infection and contamination of the aspirate but also to warrant accurate fluid analysis. ${ }^{19}$ Chronic arthritis in leprosy is characterized by finding a few numbers or no bacilli in the joint. $^{4,12}$ We referred the patient to the Department of Orthopedic and Traumatology to perform synovial fluid aspiration from the interphalangeal joint of the hand and found numerous acid-fast bacilli from acid-fast staining of the synovial material. The patient had joint destruction and subluxation, and did not show any symptoms or history of leprosy reactions. We could conclude that this patient had chronic arthritis.

The key steps involved in the management are early detection of leprosy and treatment by MDT to prevent future deformities and disabilities. ${ }^{1,4,7}$ Treatments of MB leprosy according to the World Health Organization (WHO) include a combination of rifampicin, clofazimine, and dapsone. ${ }^{1,4}$ It is important to distinguish between acute and chronic arthritis because they share similar characteristics but require a different kind of treatment and often show different responses after treatment. ${ }^{4}$ Patients with acute arthritis need glucocorticoids in addition to MDT and show improvements within a few weeks after therapy. ${ }^{1,4}$ Leprosy patients with chronic arthritis do not require additional glucocorticoids. Patients commonly achieved substantial improvements after therapy although their arthritis never resolved fully. ${ }^{4}$ Several other managements for musculoskeletal involvement in leprosy are physiotherapy, monitoring and treatment of nerve damage, and surgical treatment. ${ }^{1}$ This patient received MDT for MB leprosy, resulting in the reduction of swelling in fingers and toes after two months of treatment and improved ability to perform daily tasks.

\section{Conclusion}

Leprosy must be considered among the differential diagnoses of musculoskeletal disorders, especially in endemic regions. Arthritis due to leprosy is frequently forgotten in current clinical practices. The examination of bone and joint changes in leprosy patients may help early detection and management of musculoskeletal 
complications, to prevent future deformities and disabilities.

\section{Abbreviations}

MB, multibacillary; MDT, multidrug therapy; M. leprae, Mycobacterium leprae; RA, rheumatoid arthritis; WHO, World Health Organization.

\section{Ethical Approval}

This study was conducted in compliance with the Declaration of Helsinki, Good Clinical Practices, local regulatory requirements, and was approved by the Medical Ethics Committee of Hasan Sadikin General Hospital Bandung (approval number: LB.02.01/X.6.5/ 229/2020).

\section{Consent for Publication}

The patient signed informed consent forms. He also signed forms giving consent for the use of case details and images for publication and for scientific purposes.

\section{Acknowledgment}

Authors would like to thank all staff of the Dermatology and Venereology Department, Faculty of Medicine Universitas Padjadjaran - Hasan Sadikin General Hospital Bandung.

\section{Author Contributions}

All authors made a significant contribution to the work reported, whether that is in the conception, study design, execution, acquisition of data, analysis and interpretation, or in all these areas; took part in drafting, revising or critically reviewing the article; gave final approval of the version to be published; have agreed on the journal to which the article has been submitted; and agree to be accountable for all aspects of the work.

\section{Disclosure}

The authors report no conflicts of interest in this work.

\section{References}

1. Moonot P, Ashwood N, Lockwood D. Orthopaedic complications of leprosy. J Bone Joint Surg Br. 2005;87(10):1328-1332. doi:10.1302/ 0301-620X.87B10.16596

2. Gupta L, Zanwar A, Wakhlu A, Agarwal V. Leprosy in the rheumatology clinic: an update on this great mimic. Int $J$ Rheum Dis. 2016;19(10):941-945. doi:10.1111/1756-185X.13023

3. Alam E, Emadi SA. Case of arthritis secondary to leprosy. Springerplus. 2014;15(3):734. doi:10.1186/2193-1801-3-734

4. Chauhan S, Wakhlu A, Agarwal V. Arthritis in leprosy. Rheumatology. 2010;49(12):2237-2242. doi:10.1093/rheumatology/ keq264

5. Gupta S, Li C, Thallapally VK, Sharma P, Nahas J. Chronic hand swelling and dactylitis in leprosy: a case report and review of the literature. Cureus. 2021;13(2):e13451.

6. Rath D, Bhargava S, Kundu BK. Leprosy mimicking common rheumatoloic entities: a trial for clinician in the era of biologics. Case Rep Rheumatol. 2014;2014:429698. doi:10.1155/2014/429698

7. De Lagran ZM, Arrieta-Egurrola A, Gonzalez-Perez R, SoloetaArechavala R. Bone complications in a patient with lepromatous leprosy. Actas Dermosifiliogr. 2009;100(7):615-634.

8. Gao LN, Zhong B, Wang Y. Rheumatoid arthritis-like features in hansen disease: a case report. Medicine (Baltimore). 2018;97(29): e11590. doi:10.1097/MD.0000000000011590

9. Fernandes TR, Korinfskin JP, Espindola MM, Correa LM. Arthritis and diagnosis of leprosy: a case report and review of the literature. An Bras Dermatol. 2014;89(2):323-325. doi:10.1590/abd18064841.20142857

10. Kumar WR, Kothari SK, Swamy MKS. Deformities and bony changes in leprosy. IJPMR. 2014;25(1):13-17.

11. Ankad B, Halawar RS. Bone involvement in leprosy: early changes. Radiol Infect Dis. 2015;1(2):88-89. doi:10.1016/j.jrid.2015.02.007

12. Henriques CC. Leprosy and rheumatoid arthritis: consequence or association. BMJ Case Rep. 2012;1:1-4.

13. Agarwal V, Agarwal A. Acute and chronic bacterial infections in rheumatology practice. Indian $J$ Rheumatol. 2011;6(1):69-74. doi:10.1016/S0973-3698(11)60035-6

14. Gibson T, Ahsan Q, Hussein K. Arthritis of leprosy. Br J Rheumatol. 1994;33(10):963-966. doi:10.1093/rheumatology/33.10.963

15. Atkin SL, el-Ghobarey A, Kamel M, Owen JP, Dick WC. Clinical and laboratory studies of arthritis in leprosy. BMJ. 1989;298 (6685):1423-1425. doi:10.1136/bmj.298.6685.1423

16. Atkin SL, Welbury RR, Stanfield E, Beavis D, Iwais B, Dick WC. Clinical and laboratory studies of inflammatory polyarthritis in patients with leprosy in Papua New Guinea. Ann Rheum Dis. 1987;46(9):688-690. doi:10.1136/ard.46.9.688

17. Cossermelli-Messina W, Festa Neto C, Cossermelli W. Articular inflammatory manifestations in patients with different forms of leprosy. J Rheumatol. 1998;25(1):111-119.

18. Slim FJ, Faber WR, Maas M. The role of radiology in nerve function impairment and its musculoskeletal complications in leprosy. Lepr Rev. 2009;80(4):373-387. doi:10.47276/1r.80.4.373

19. Seidman AJ, Limaiem F. Synovial fluid analysis. In: StatPearls [Internet]. Treasure Island (FL): StatPearls Publishing; 2020. 


\section{Publish your work in this journal}

The International Medical Case Reports Journal is an international, peer-reviewed open-access journal publishing original case reports from all medical specialties. Previously unpublished medical posters are also accepted relating to any area of clinical or preclinical science. Submissions should not normally exceed 2,000 words or 4 published pages including figures, diagrams and references. The manuscript management system is completely online and includes a very quick and fair peer-review system, which is all easy to use. Visit http://www.dovepress.com/testimonials.php to read real quotes from published authors. 